\title{
Circular RNA Expression Profile in Laryngeal Squamous Cell Carcinoma Revealed by Microarray
}

\author{
Yue Fan Xin Xia Yingying Zhu Wenwen Diao Xiaoli Zhu Zhiqiang Gao \\ Xingming Chen
}

Department of Otolaryngology-Head and Neck Surgery, Peking Union Medical College Hospital, Peking Union Medical College and Chinese Academy of Medical Sciences, Beijing, China

\section{Key Words}

CircRNAs $\bullet$ Laryngeal squamous cell carcinoma $\bullet$ Microarray $\bullet$ miRNA sponges $\bullet$ Bioinformatics analysis

\begin{abstract}
Background/Aims: A growing body of evidence has suggested that circular RNAs (circRNAs) have crucial functions in the regulation of gene expression, and their dysregulation has been implicated in various types of cancers. However, the roles of circRNAs in laryngeal cancer remain largely unknown. This study investigated the global changes in the expression pattern of circRNAs in laryngeal squamous cell carcinoma (LSCC) to identify potential differentially expressed circRNAs. Methods: Microarray-based circRNA expression was determined in LSCC and paired normal laryngeal tissues. Pathway analyses of the genes producing differentially expressed circRNAs were performed to predict the function of circRNAs using standard enrichment computational methods. Expression levels of candidate circRNAs and microRNAs (miRNAs) were detected by quantitative real-time PCR. The circRNA/ miRNA interactions were constructed using bioinformatics methods to predict the binding of miRNA with circRNA. Results: We identified 506 differentially expressed circRNAs from human LSCC and normal laryngeal mucosa tissues. We confirmed that hsa_circ_0044520 and hsa_circ_0044529 were significantly upregulated in LSCC tissues. The most likely potential target miRNAs for hsa circ_0044520 and hsa_circ_0044529 were hsa-miR-4726-5p and hsa-miR-4640-5p, respectively. Functional analysis showed that hsa_circ_0044520 and hsa_circ_0044529 were involved in the process of collagen synthesis. Conclusion: Competitive endogenous RNA network prediction and bioinformatics functional analysis revealed that hsa_circ_0044520 and hsa_circ_0044529 play important regulatory roles by sponging hsa-miR-4726-5p and hsa-miR-4640-5p, thereby providing novel insights into the tumorigenesis of LSCC.

Y. Fan and X. Xia contributed equally to this work.

\begin{tabular}{ll}
\hline Prof. Xingming Chen & Department of Otolaryngology-Head and Neck Surgery, Peking Union Medical College Hospital \\
& No. 1, Shuaifuyuan, Wangfujing, Beijing 100730 (China) \\
& Tel. +86-10-6915-6328, Fax+86-10-6915-6311, E-Mail xingming.chen@hotmail.com
\end{tabular}
\end{abstract}




\section{Introduction}

Laryngeal cancer is one of the most common malignant neoplasms of the head and neck, accounting for $1 \%$ of cancer-related deaths worldwide [1]. A report from the National Central Cancer Registry of China stated that there were approximately 26, 400 new laryngeal cancer cases and 14, 500 deaths from laryngeal cancer in 2015, and the morbidity and mortality have been increasing in recent years [2]. Approximately $60 \%$ of patients present with advanced disease (stage III or IV) at diagnosis, and these patients can experience dysphonia, dyspnea, and dysphagia, which cause serious psychological distress and severely affect the quality of life [3,4]. Although considerable progress has been made in surgical techniques and adjuvant chemotherapies, laryngeal cancer is unfortunately one of a few oncologic diseases in which the 5-year survival rate has decreased in the past 40 years, from $66 \%$ to $63 \%[5,6]$. Therefore, the identification of new biomarkers associated with the tumorigenesis of laryngeal cancer has critical significance for the early diagnosis and treatment of this disease.

Circular RNAs (circRNAs) represent a new class of abundant and stable endogenous noncoding RNAs that participate in the pathogenesis of malignant tumors [7-9]. Most known circRNA coding sequences are located in protein coding genes and thus show some general characteristics of most genes, such as conservation among species and temporal and spatial specificity of expression profiles [10,11]. circRNAs do not form covalently closed loop structures with $5^{\prime}$ or $3^{\prime}$ polarities or polyadenylated tails, which renders them more stable than linear transcripts [12-14]. Recently, they were found to sequester microRNAs (miRNAs) by recognition of miRNA response elements (MREs). Most identified circRNAs can sequester multiple miRNAs, and thus might dysregulate the downstream miRNA-targeted transcripts in many eukaryotes [15].

Although the biological functions of circRNAs remain to be elucidated, many reports have identified a relationship between circRNAs and oncogenesis. Expression analyses of various tumor cell lines showed widespread expression of ciRS-7 in neuroblastomas, astrocytoma and lung carcinomas [16]. Zhao et al. illustrated that clusters of circRNAs, such as hsa_circ_404833 and hsa_circ_406483, were aberrantly expressed in early stage lung adenocarcinoma [17]. Li et al. revealed that a number of circRNAs, such as hsa_circ_0001946 and hsa_circ_0005397, were dysregulated in pancreatic cancer [18]. Liu et al. identified the comprehensive expression profile of circRNAs in osteosarcoma, and confirmed that hsa_circ_103801 and hsa_circ_104980 might be involved in the initiation and progression of osteosarcoma [19]. These reported associations between circRNA expression and various cancers indicate their potential use as diagnostic biomarkers in cancer.

Despite the promising link between circRNA expression and human cancers, the role of circRNAs in laryngeal cancer remains largely unknown. We hypothesized that dysregulation of circRNAs might be involved in laryngeal tumorigenesis. Here, we used a high-throughput microarray platform to evaluate circRNA expression profiles in laryngeal squamous cell carcinoma (LSCC) and normal laryngeal mucosa from 5 LSCC patients and 5 cancer-free patients, respectively. Then, we performed step-wise bioinformatics analysis and quantitative real-time polymerase chain reaction (qRT-PCR) for validation. Our data provide a novel basis for the functional role of circRNAs in laryngeal cancer.

\section{Materials and Methods}

Ethics statement

This study was approved and supervised by The Ethics Committee of Peking Union Medical College Hospital (Beijing, China). Written informed consent was obtained from patients for research purposes. 


\section{Cellular Physiology Cell Physiol Biochem 2018;50:342-352

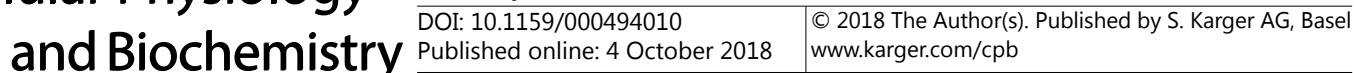

Fan et al.: Circular RNA Expression and Laryngeal Cancer

\section{Patients and tissue specimens}

In total, 35 patients with newly diagnosed, histopathologically confirmed and untreated LSCC were consecutively recruited at Peking Union Medical College Hospital from January 2013 to December 2015. Patients were excluded if they had a second primary tumor, metastasis, or a histopathologic diagnosis other than squamous cell carcinoma. Also, 35 cancer-free patients with laryngeal trauma who were admitted to the same hospital during the same period were recruited as controls. Pathological results were available for all individuals in both groups and the results were reviewed by an experienced pathologist. None of the patients had a previous history of cancer or had received chemotherapy or radiation therapy prior to surgery. Five LSCC cases and 5 cancer-free controls were selected for microarray assay, and the demographic and clinical characteristics are presented in Table 1.

The excised tissue specimens were snap-frozen in liquid nitrogen immediately after resection and stored at $-80^{\circ} \mathrm{C}$ until RNA was extracted.

\section{RNA extraction and quality control}

Total RNA was isolated from frozen tissues in liquid nitrogen using TRIzol reagent (Invitrogen, Carlsbad, CA, USA) according to the manufacturer's instructions. RNA quantity and quality were evaluated with OD260/280 readings obtained using the Nano Drop ND-1000 spectrophotometer (NanoDrop Technologies/ Thermo Scientific, Wilmington, DE, USA) and RNA integrity was assessed by $1 \%$ formaldehyde denaturing gel electrophoresis.

\section{RNA digestion, amplification, labeling, and hybridization}

Total extracted RNA was treated with Rnase R (Epicentre, Madison, WI, USA) to remove linear RNA. Enriched circRNAs were amplified and then transcribed into fluorescent circRNAs using random primers according to the instructions for the CapitalBio cRNA Amplification and Labeling Kit (CapitalBio, Beijing, China). Amplified circRNA was purified with the RNeasy Mini Kit (Qiagen, Dusseldorf, Germany). Concentration and specific activity of the labeled circRNAs were assessed using the Nano Drop ND-1000. The labeled circRNAs were then hybridized onto the Agilent human circRNA array (version 1.0; Agilent Technologies, Santa Clara, CA, USA). The slides were rotated at $20 \mathrm{rpm}$ at $42^{\circ} \mathrm{C}$ in an Agilent hybridization oven overnight and were then washed and scanned using the Agilent G2505C Scanner.

\section{Microarray assay and data analysis}

The Arraystar Human Circular RNA Microarray V2.0S (815k, Arraystar Inc., Rockville, MD, USA), with a total of 142, 404 circRNA probes on the microarray, was used to assess the differential expression of circRNAs in LSCC versus normal mucosa samples. Raw data were extracted from the scanned images by Agilent Feature Extraction software (version 11.0.1.1). GeneSpring software (version 13.0; Agilent Technologies) was used for the microarray data analysis, including data summarization, normalization, and quality control. The workflow was performed according to the manufacturer's instructions. Three quality control indicators were used to filter out most false signals of the microarray: 1) $P<0.001,2$ ) sample detection rate $>50 \%$, and 3 ) fold change in expression $>2.0$ between the LSCC and normal mucosa tissue samples. Interaction analysis of circRNA/miRNA was performed using Arraystar's software, which is based on miRanda and TargetScan. Cytoscape software was used for constructing the circRNA-miRNA networks. Kyoto Encyclopedia of Genes and Genomes (KEGG) pathway enrichment analysis was performed by using the KEGG orthology-based annotation system. 


\section{Cellular Physiology Cell Physiol Biochem 2018;50:342-352

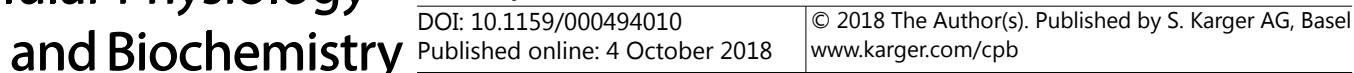

Fan et al.: Circular RNA Expression and Laryngeal Cancer

Quantitative real-time PCR ( $q R T-P C R$ )

Extracted RNA was subjected to cDNA synthesis using the SuperScript ${ }^{\mathrm{TM}}$ III Reverse Transcriptase Kit (Invitrogen), according to the manufacturer's instructions. qRT-PCR was performed using the qPCR Master Mix (Arraystar) on a ViiA 7 Real-time PCR System (Applied Biosystems) following the manufacturer's instructions. All primers used in this study are listed in Table S1 for all supplementary material see www. karger.com/ 10.1159/000494010/). miRNA was converted to cDNA using the Stem-Loop miRNA Reverse Transcription (Ribobio, Guangzhou, China) with specific primers for hsamiR-4726-5p, hsa-miR-4640-5p, and U6 snRNA as a reference control.

\section{Statistical analysis}

Data are presented as the mean \pm standard error of the mean (SEM), and were analyzed by SPSS (SPSS for Windows version 21.0; SPSS, Chicago, IL, USA). Artworks were created by using GraphPad Prism 5.0 (GraphPad Software, La Jolla, CA). Wilcoxon matched pairs signed-rank test was used to compare the circRNA expression levels in LSCC versus normal mucosa tissues. Average cycle thresholds for the technical triplicates were calculated to yield 1 value per primer set for each biological replicate. Normalization was performed using GAPDH or U6 with the formula $2^{-\Delta \Delta \mathrm{CT}}$ to determine relative expression. Averages and standard deviations of the normalized biological replicate values were plotted in the figures and used in t-test calculations. $P<0.05$ was considered statistically significant.

\section{Results}

Global profiling of circRNAs in LSCC tissues

We performed microarray analysis in a cohort of 5 pairs of LSCC and normal tissues, and identified a total of 82 , 275 circRNAs including 3, 932 differentially expressed and 78, 343 non-differentially expressed circRNAs. After the data filtering,

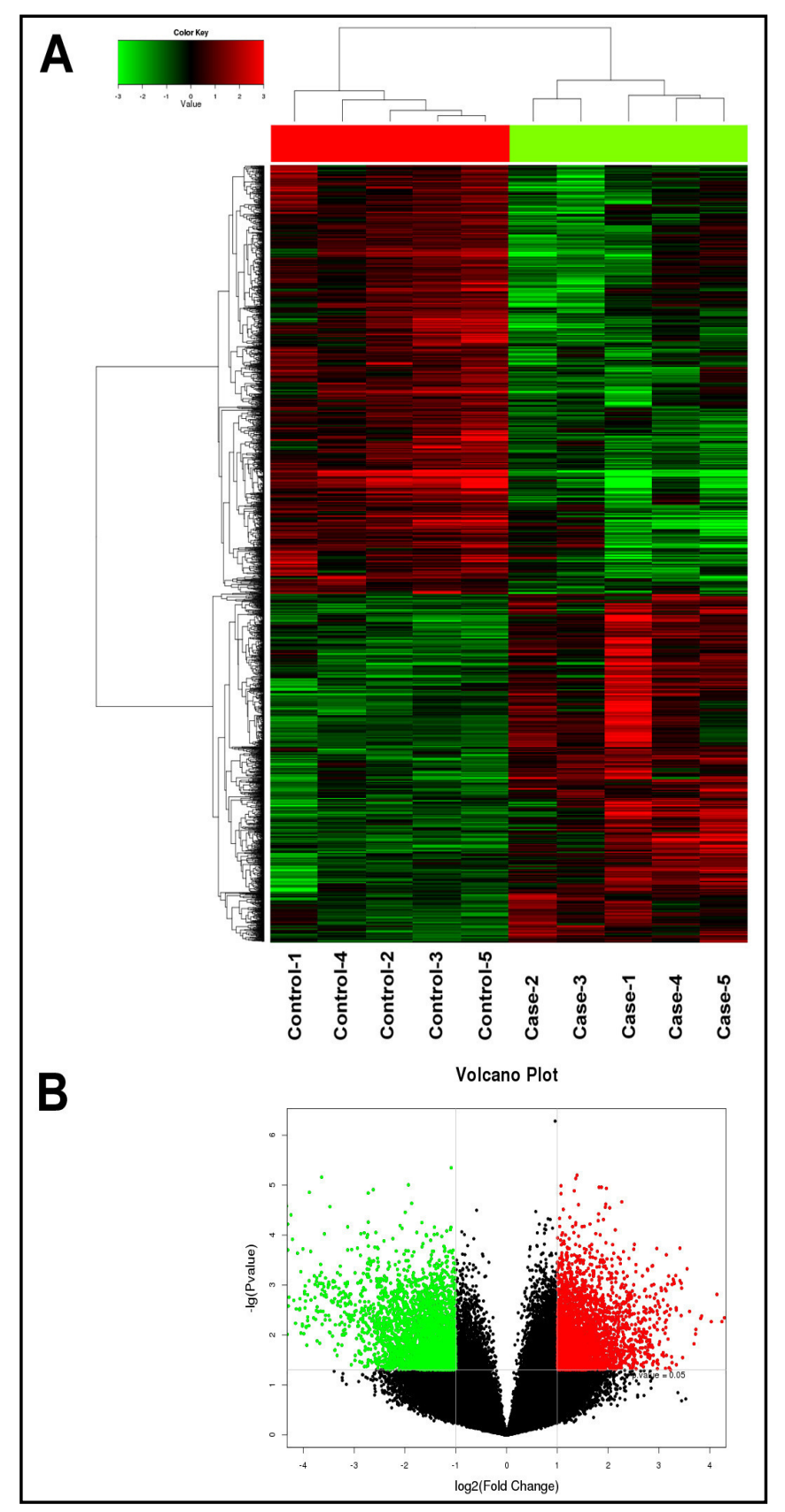

Fig. 1. Hierarchical clustering and volcano plots demonstrate the differentially expressed circRNAs in LSCC tissues compared with paired normal tissues. (A) Hierarchical clustering analysis for 10 samples in the case group (Case-1, Case-2, Case-3, Case-4, and Case-5) and control group (Control-1, Control-2, Control-3, Control-4 and Control-5). (B) Differentially expressed circRNAs are displayed by volcano plots. The green and red sections indicate $>2$-fold decreased and increased expression of the dysregulated circRNAs in LSCC tissues, respectively $(\mathrm{P}<0.05)$. 
Table 2. The top 10 upregulated and downregulated differentially expressed circRNAs in LSCC tissues compared to those in normal laryngeal tissues. Abbreviation: FC, fold change; chr, chromosome; MRE, microRNA response element. *No. max MRE: The maximum number of a microRNA response element for the circRNA

\begin{tabular}{|c|c|c|c|c|c|c|}
\hline circRNA ID & $P$ value & $\mathrm{FC}$ & chr & Gene symbol & No. miRNA targets & No. $\max$ MRE \\
\hline \multicolumn{7}{|c|}{ Top 10 upregulated circRNAs } \\
\hline hsa_circ_0004507 & 0.009 & 2.231 & chr19 & SAE1 & 0 & 0 \\
\hline hsa_circ_0001883 & 0.009 & 3.072 & chr9 & ORM1 & 324 & 5 \\
\hline hsa_circ_0022373 & 0.009 & 3.888 & chr11 & FADS1 & 16 & 3 \\
\hline hsa_circ_0020377 & 0.009 & 3.574 & chr10 & ADAM12 & 0 & 0 \\
\hline hsa_circ_0076130 & 0.009 & 2.173 & chr6 & FANCE & 130 & 6 \\
\hline hsa_cir__0060211 & 0.009 & 2.363 & chr20 & KIAA0889 & 382 & 8 \\
\hline hsa_circ_0075264 & 0.009 & 3.578 & chr5 & ADAMTS2 & 8 & 2 \\
\hline hsa_circ_0057440 & 0.009 & 3.304 & chr2 & COL5A2 & 28 & 3 \\
\hline hsa_circ_0091851 & 0.009 & 3.031 & $\operatorname{chrX}$ & FLNA & 502 & 8 \\
\hline hsa_circ_0092012 & 0.009 & 2.092 & $\operatorname{chr} x$ & SAE1 & 45 & 4 \\
\hline \multicolumn{7}{|c|}{ Top 10 downregulated circRNAs } \\
\hline hsa_cir__0037362 & $<0.001$ & 120.657 & chr16 & C16orf73 & 0 & 0 \\
\hline hsa_cir__0020763 & $<0.001$ & 112.837 & chr11 & None & 623 & 7 \\
\hline hsa_circ_0037361 & $<0.001$ & 111.193 & chr16 & C16orf73 & 0 & 0 \\
\hline hsa_circ_0077886 & $<0.001$ & 96.224 & chr6 & EYA4 & 0 & 0 \\
\hline hsa_circ_0077887 & $<0.001$ & 52.249 & chr6 & EYA4 & 8 & 3 \\
\hline hsa_cir__0020758 & $<0.001$ & 35.563 & chr11 & None & 513 & 8 \\
\hline hsa_circ_0077884 & $<0.001$ & 32.025 & chr6 & EYA4 & 9 & 2 \\
\hline hsa_circ_0084765 & $<0.001$ & 29.394 & chr8 & EYA1 & 4 & 2 \\
\hline hsa_circ_0020754 & $<0.001$ & 26.617 & chr11 & None & 100 & 6 \\
\hline hsa_circ_0020753 & $<0.001$ & 25.489 & chr11 & None & 43 & 4 \\
\hline
\end{tabular}

Table 3. The 21 host genes and the number of related circRNAs involved in collagen formation pathway

\begin{tabular}{|c|c|c|c|}
\hline $\begin{array}{l}\text { Representative circRNAs } \\
\text { (incomplete) }\end{array}$ & Number of circRNAs & Regulation of circRNAs & Host Gene Symbol \\
\hline hsa_circ_0044529, hsa_circ_0044520 ... & 45 & up & COL1A1 \\
\hline hsa_circ_0057366,hsa_circ_0057405 ... & 23 & up & COL3A1 \\
\hline hsa_circ_0081168,hsa_circ_0081160 ... & 22 & up & COL1A2 \\
\hline hsa_circ_0030867,hsa_circ_0030854 ... & 18 & up & COL4A2 \\
\hline hsa_circ_0065428,hsa_circ_0065411 ... & 11 & up & COL7A1 \\
\hline hsa_circ_007 7067,hsa_circ_0077064 ... & 10 & up & COL12A1 \\
\hline hsa_circ_0057440, hsa_circ_0057424 ... & 3 & up & COL5A2 \\
\hline hsa_circ_0030837, hsa_circ_0030838 ... & 2 & up & COL4A1 \\
\hline hsa_circ_0089440, hsa_circ_0089461 ... & 2 & up & COL5A1 \\
\hline hsa_circ_0058826, hsa_circ_0058840 ... & 2 & up & COL6A3 \\
\hline hsa_circ_0066633 & 1 & up & COL8A1 \\
\hline hsa_circ_0088168 & 1 & up & COL27A1 \\
\hline hsa_circ_0085417 & 1 & down & COL14A1 \\
\hline hsa_circ_0009884, hsa_circ_0009897 ... & 4 & up & PLOD1 \\
\hline hsa_circ_0075264, hsa_circ_0006826 ... & 3 & up & ADAMTS2 \\
\hline hsa_circ_0015602, hsa_circ_0015601 ... & 2 & up & LAMC2 \\
\hline hsa_circ_0083670, hsa_circ_0083669 ... & 2 & up & LOXL2 \\
\hline hsa_circ_0057085 & 1 & up & ITGA6 \\
\hline hsa_circ_0047257 & 1 & up & LAMA3 \\
\hline hsa_circ_0060571 & 1 & up & MMP9 \\
\hline hsa_circ_0023628 & 1 & up & SERPINH1 \\
\hline
\end{tabular}

506 circRNA reads were significantly differentially expressed in LSCC tissues, of which 386 were upregulated and 120 were downregulated (Table S2). Hierarchical clustering (Fig. 1A) and volcano plots (Fig. 1B) revealed that the expression profiles of circRNAs between LSCC and matched control normal tissues were diverse. The top 10 upregulated and downregulated circRNAs sorted by their fold change values (absolute value) are listed in Table 2.

Pathway analysis of the genes producing differentially expressed circRNAs

To determine the pathways in which the differentially expressed circRNAs were involved, KEGG pathway analysis was performed to analyze the genes that produced them. KEGG analyses indicated that the differentially expressed circRNAs were related to the process of collagen synthesis. A total of 21 genes were identified in this pathway, of which 13 were members of the collagen family of genes (Table 3). Disease enrichment analysis suggested that the abnormal expression of circRNAs in LSCC was associated with EhlersDanlos syndrome (a congenital connective tissue hypoplasia synthesis syndrome), which is related to collagen synthesis abnormality [20]. Fig. 2 shows the top 20 significantly enriched pathway terms and disease terms.

\section{Candidate selection and construction of the circRNA-miRNA network}

circRNAs can sequester relevant miRNAs with MREs and thus post-transcriptionally regulate downstream miRNA-target genes [15]. Therefore, we used a circRNA-miRNA network strategy for candidate molecule selection. First, a miRanda-based software was used to scan the entire sequences of the selected circRNAs, after which all of the possible miRNA binding sites were recorded. The screening conditions for the circRNA-miRNA interactions were as follows: the candidate miRNA seed sequence needed to exactly match 


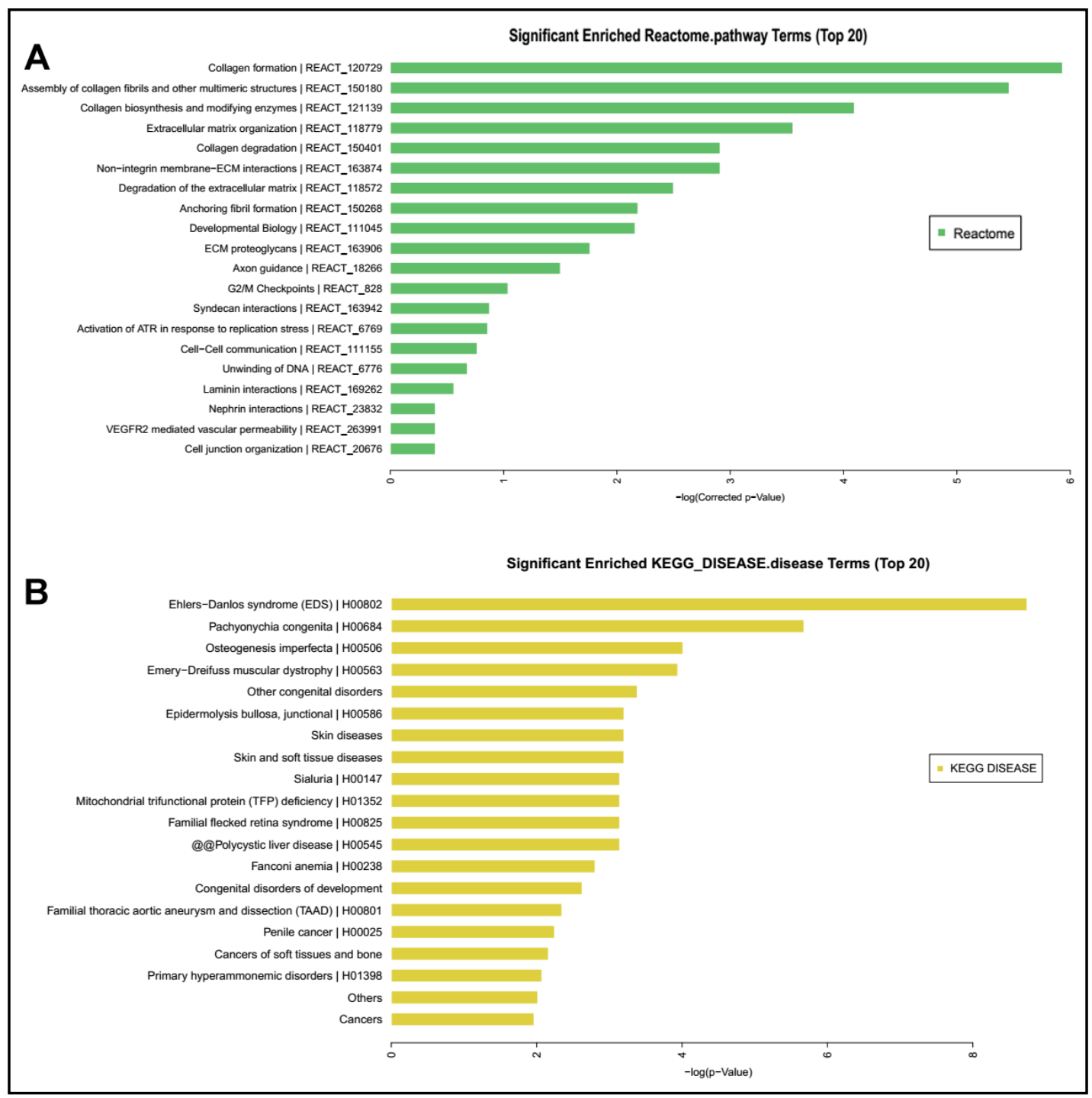

Fig. 2. Results of KEGG pathway and disease enrichment analyses. (A) Top 20 classes of KEGG pathway enrichment terms. (B) Top 20 disease enrichment terms.

its host circRNA, and the host circRNA had to have more than 10 MREs of a single miRNA to avoid false matches. The top 20 upregulated and downregulated candidate circRNAs with the largest number of MREs were selected (Table S3). The top 20 target miRNAs captured by multiple circRNAs are also listed (Table S4). Because each circRNA has multiple miRNA binding sites, and each miRNA can be captured by different circRNAs, we ranked these candidates according to circRNA coupling numbers for miRNAs and MRE number for circRNAs. These two sets of data were intersected, and the top 10 ranked upregulated and top 5 ranked downregulated candidate circRNAs were identified (Table 4). Based on these dysregulated circRNAs and their predicted MREs, a network map of circRNA-miRNA interactions with Cytoscape was established (Fig. S1). Interestingly, the overlapped data in the upregulated circRNAs were focused on the collagen, type 1, alpha 1 (COL1A1) gene and COL7A1 gene, which were in accordance with the results of the bioinformatics analysis. The downregulated circRNAs was focused on the LPHN2 gene and an intergenic region on chromosome 11. 
Evaluation of circRNA expression by qRT-PCR analysis

Next, we used qRT-PCR to examine the levels of the top 10 ranked upregulated and top 5 ranked downregulated candidate circRNAs that were identified above in the test cohort. None of the downregulated circRNAs in the microarray could be detected by qRT-PCR. Among the upregulated circRNAs, only hsa_circ_0044519, hsa_circ_0044520, hsa_circ_0044529, and hsa_circ_0065404 showed significantly higher expression in LSCC tissues (Fig. 3; $\left.{ }^{*} P<0.05,{ }^{* *} P<0.01\right)$. These four circRNAs were then further analyzed in an independent validation cohort with 30 LSCC patients and 30 cancer-free controls. The expression patterns of hsa_circ_0044520 and hsa_circ_0044529 were consistent with those in the test cohort (Fig. 4 ; ${ }^{*} P<0.05,{ }^{* *} P<0.01$ ), whereas the expression of hsa_circ_004519 and hsa_circ_0065404 showed no significant difference between the two groups (Fig. S2).

\section{Evaluation of the target miRNAs of hsa_circ_0044520 and hsa_circ_0044529 in LSCC \\ After qRT-PCR analysis,} only hsa_circ_0044520 and hsa_circ_0044529 showed significantly higher levels in LSCC and captured two target miRNAs on our list, hsa-miR-4726-5p and hsa-miR-4640-5p. Their expression levels were found to be significantly lower in LSCC tissues by qRT-PCR analysis in all 35 paired samples (Fig. 5; $\left.{ }^{* * *} P<0.001\right)$. Further analysis was performed using TargetScan (http://www.targetscan.org/) for miRNA target gene scanning and DAVID (https://david.ncifcrf. gov/) for functional annotation. Interestly, we found that target genes for hsa-miR-4726-5p and hsa-miR-4640-5p were most enriched in "Tobacco Use Disorders" (Tables S5 and S6).
Table 4. Top 10 ranked upregulated and top 5 ranked downregulated candidate circRNAs

\begin{tabular}{lcc}
\hline Corresponding circular RNAs & Gene Symbol & \multicolumn{1}{c}{ Chromosomal Location } \\
\hline Upregulated circRNAs & & \\
hsa_circ_0005534 & ERC1 & chr12:1107489-1137738 \\
hsa_circ_0044519 & COL1A1 & chr17:48263677-48275146 \\
hsa_cir__0044518 & COL1A1 & chr17:48263677-48273560 \\
hsa_circ_0044520 & COL1A1 & chr17:48264375-48273337 \\
hsa_circ_0044517 & COL1A1 & chr17:48263677-48263677 \\
hsa_circ_0044529 & COL1A1 & chr17:48266102-48277308 \\
hsa_cir__0065404 & COL7A1 & chr3:48602842-48630132 \\
hsa_circ_0044527 & COL1A1 & chr17:48266102-48273560 \\
hsa_circ_0044539 & COL1A1 & chr17:48266737-48273728 \\
hsa_circ_0029399 & GLT1D1 & chr12:129373183-129411699
\end{tabular}

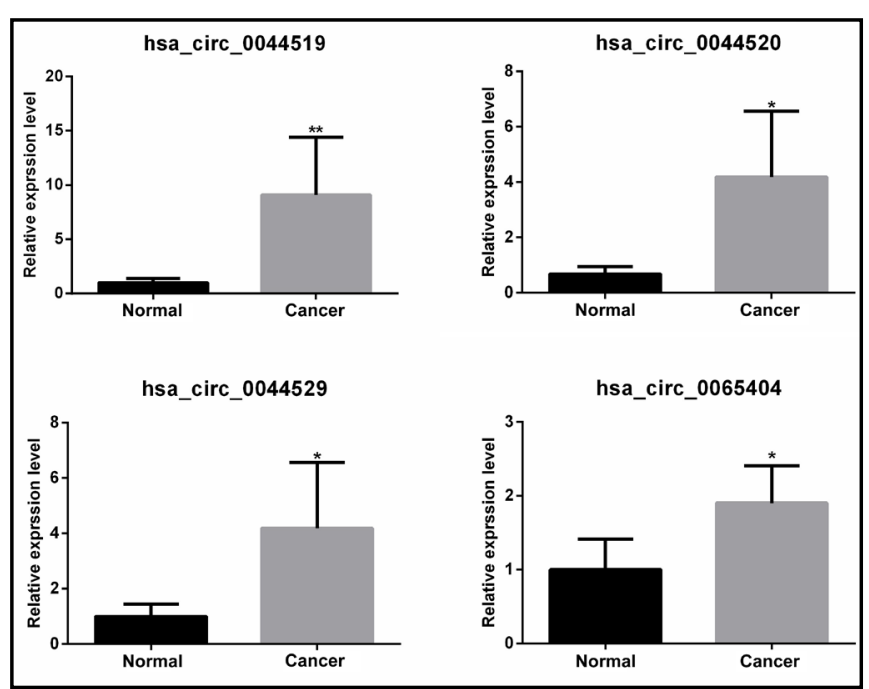

Fig. 3. Relative expression levels of hsa_circ_0044519, hsa circ_0044520, hsa_circ_0044529, and hsa_circ_0065404 in five LSCC tissues and paired normal control tissues in the test cohort.

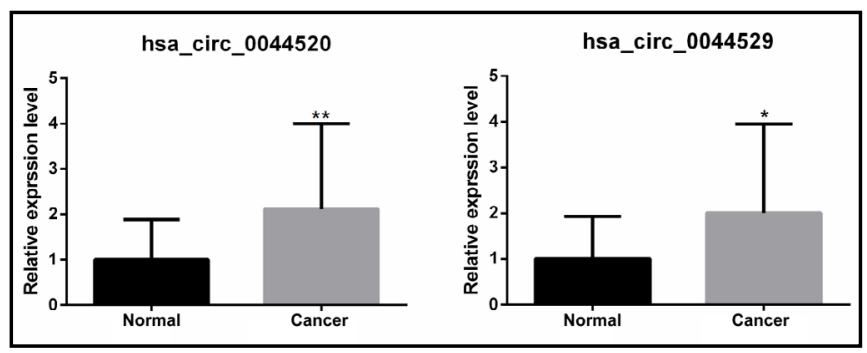

Fig. 4. Relative expression levels of hsa_circ_0044520 and hsa_ circ_0044529 in 30 LSCC tissues and normal control tissues in the validation cohort. 
Discussion

circRNAs are a class of endogenous noncoding RNAs with highly conserved sequences and a high degree of stability in cells. Recent studies have found that circRNAs can serve as gene regulators through a "circRNAmiRNA-mRNA" regulatory system [7]. This novel epigenetic regulatory mechanism may be crucial in physiological and pathological processes. While numerous reports have highlighted aberrant expression of circRNAs in human cancer, their expression and roles in LSCC remain to be determined.

Studies on the expression of circRNAs in cancer have involved tumor tissue and adjacent normal tissue, which may not be ideal for demonstrating of the role of circRNAs in tumorigenesis, because the differentially expressed circRNAs between the tissues could result in tumorigenesis. In addition, previous studies have found aberrant gene expression in cells comprising normal adjacent tissue, which could have important implications in cancer prognosis and progression $[21,22]$. Thus, in this study, normal laryngeal mucosa samples from patients with laryngeal trauma were used as controls. We performed genomewide circRNA profiling in LSCC tissues using microarray analysis and identified 3, 932 dysregulated circRNAs. By applying a set of bioinformatics tools, we found that the most upregulated expressed circRNAs were mainly associated with the collagen family genes.

To identify the most informative candidates, we designed a "circRNA-miRNA interacting network"-based strategy, based on the hypothesis that the functional dysregulated circRNAs should be potent in capturing target miRNAs. We focused on the top 10 upregulated and top 5 downregulated circRNAs. After qRT-PCR of both the discovery cohort and an independent validation cohort, hsa_circ_0044520 and hsa_circ_0044529 were found to be significantly higher expressed in LSCC tissues than in the normal controls.

circRNAs share the same sequence as their corresponding linear isomers but are formed through independent splicing mechanisms [23]. Both hsa_circ_0044520 and hsa circ 0044529 are hosted in the COL1A1 gene, which agrees with the result of the bioinformatics analysis. COL1A1 encodes the subunit of type I collagen, which is preferentially synthesized in the bone, dermis, and tendons [24], and is recognized as a tumor-related gene. Previous studies reported that COL1A1 is upregulated in the tumor tissues in breast, gastric and liver cancers, and most likely participates in tumorigenesis [25-27]. Thus, the mis-splicing of COL1A1 may play a potential role in LSCC tumorigenesis.

Many studies have established a wide role for miRNAs in most biological processes and in cancer initiation and progression [28-30]. circRNAs can function as miRNA "sponges" through MREs, which naturally sequester and competitively suppress the activity of miRNAs $[9,15]$. This competitive endogenous RNA network may further disturb the downstream gene expression profiles. In this study, we used bioinformative analysis to identify hsamiR-4726-5p as a target miRNA for hsa_circ_0044520, and hsa-miR-4640-5p as a target for hsa_circ_0044529. Then we evaluated the expression levels of these two miRNAs, and found that both were significantly lower in LSCC tissues than in normal control tissues. Further bioinformatics analysis revealed that target genes for hsa-miR-4726-5p and hsa-miR-4640$5 p$ were most enriched in "Tobacco Use Disorders". This category of disorders contains numerous oncogenes related with tumorigenesis such as activating transcription factor 3 , which is involved in the progression of LSCC, and as such, may provide clinical information for the evaluation of LSCC prognosis [31]. As prior studies have indicated that tobacco use is a strong risk factor for numerous cancers and shows a linear association with the development of laryngeal cancer [32,33], our results highlight the potential role of hsa_circ_0044520 and 
hsa_circ_0044529, and their regulatory networks in LSCC. We hypothesized that there was an interaction model of circRNAs in LSCC, namely the damage caused by smoking and other risk factors may disrupt the splicing pattern of COL1A1 and generate more specific circRNAs. These extra circRNAs could stably exist in cells for a long period of time, and thus block their target miRNAs such as hsa-miR-4726-5p and hsa-miR-46405p. Downstream oncogenes might be activated and initiate tumorigenesis (Fig. 6).

One limitation of this study was that all of the downregulated circRNAs found in microarrays were not detected by qRT-PCR analysis. It is possible that there were trace amounts of these circRNAs that were under the limit of qRTPCR detection, and that more sensitive detection techniques are required.

\section{Conclusion}

Our study revealed the circRNA expression signatures of LSCC. Cancer-related pathways for hsa_circ_0044520 and hsa_circ_0044529 and their target miRNAs hsa-miR-4726-5p and hsa-miR-4640-5p were constructed using bioinformatics analysis. These findings suggest that dysregulation of hsa_circ_0044520 and hsa_circ_0044529 might be involved in the pathogenesis of LSCC. In the future, a larger cohort should be examined to confirm our findings and determine whether these factors can serve as novel biomarkers for LSCC diagnosis and whether their expression levels are associated with clinicopathological characteristics.

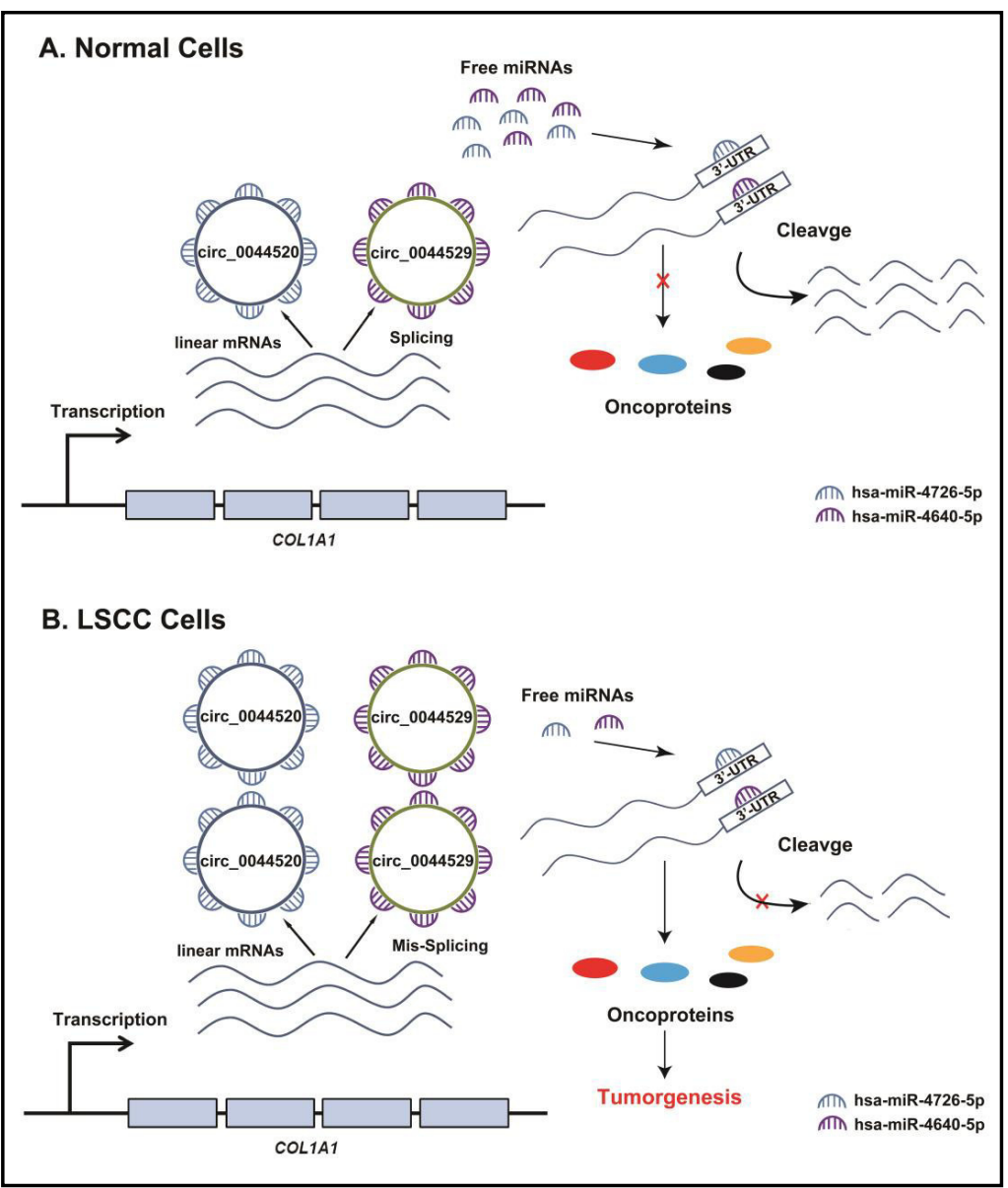

Fig. 6. Schematic diagram of the possible mechanisms for hsa_circ_0044520 and hsa_circ_0044529 regulation in the tumorigenesis of LSCC. (A) In normal laryngeal mucosa cells, hsa_circ_0044520 and hsa_circ_0044529 generated from COL1A1 functioned as miRNA "sponges" to sequester and competitively suppress the activity of hsa-miR-4726-5p and hsa-miR-4640-5p. hsa-miR4726-5p and hsa-miR-4640-5p suppressed the expression of their target RNAs, which included some oncogenes. The balance between the amount of miRNAs and their targets was well maintained. (B) In LSCC cells, the damage caused by smoking and other risk factors may disrupt the splicing pattern of COL1A1 and generate more hsa_circ_0044520 and hsa_circ_0044529. The extra circRNAs suppressed the amount of hsa-miR-4726-5p and hsa-miR$4640-5 p$, resulting in the upregulation of miRNA-regulated target oncogenes, and subsequent tumorigenesis. 


\section{Cellular Physiology Cell Physiol Biochem 2018;50:342-352 \begin{tabular}{l|l|l} 
and Biochemistry & $\begin{array}{l}\text { DOI: 10.1159/000494010 } \\
\text { Published onlIne: 40ctober } 2018\end{array}$ & $\begin{array}{l}\text { C) } 2018 \text { The Author(s). Published by S. Karger AG, Basel } \\
\text { www.karger.com/cpb }\end{array}$ \\
\hline
\end{tabular}}

\section{Acknowledgements}

This work was supported by the National Natural Science Foundation of China (Grant No 81273173).

\section{Disclosure Statement}

The authors have no conflicts of interest to disclose.

\section{References}

1 International Agency for Research on Cancer: GLOBOCAN 2012: Estimated Cancer Incidence, Mortality and Prevalence Worldwide in 2012 v1.0. URL: http://globocan.iarc.fr.

-2 Chen W, Zheng R, Baade PD, Zhang S, Zeng H, Bray F, Jemal A, Yu XQ He J: Cancer statistics in china, 2015. CA Cancer J Clin 2016;66:115-132.

-3 Groome PA, O'Sullivan B, Irish JC, Rothwell DM, Schulze K, Warde PR, Schneider KM, Mackenzie RG, Hodson DI, Hammond JA, Gulavita SP, Eapen LJ, Dixon PF, Bissett RJ, Mackillop WJ: Management and outcome differences in supraglottic cancer between ontario, canada, and the surveillance, epidemiology, and end results areas of the united states. J Clin Oncol 2003;21:496-505.

-4 Luo J, Wu J, Lv K, Li K, Wu J, Wen Y, Li X, Tang H, Jiang A, Wang Z, Wen W, Lei W: Analysis of postsurgical health-related quality of life and quality of voice of patients with laryngeal carcinoma. Medicine (Baltimore) 2016;95:e2363.

5 Rudolph E, Dyckhoff G, Becher H, Dietz A, Ramroth H: Effects of tumour stage, comorbidity and therapy on survival of laryngeal cancer patients: a systematic review and a meta-analysis. Eur Arch Otorhinolaryngol 2011;268:165-179.

6 Siegel RL, Miller KD, Jemal A: Cancer statistics, 2016. CA Cancer J Clin 2016;66:7-30.

7 Qu S, Yang X, Li X, Wang J, Gao Y, Shang R, Sun W, Dou K, Li H: Circular rna: a new star of noncoding rnas. Cancer Lett 2015;365:141-148.

8 Jeck WR, Sorrentino JA, Wang K, Slevin MK, Burd CE, Liu J, Marzluff WF, Sharpless NE: Circular rnas are abundant, conserved, and associated with alu repeats. RNA 2013;19:141-157.

-9 Memczak S, Jens M, Elefsinioti A, Torti F, Krueger J, Rybak A, Maier L, Mackowiak SD, Gregersen LH, Munschauer M, Loewer A, Ziebold U, Landthaler M, Kocks C, le Noble F, Rajewsky N: Circular rnas are a large class of animal rnas with regulatory potency. Nature 2013;495:333-338.

10 Li Z, Huang C, Bao C, Chen L, Lin M, Wang X, Zhong G, Yu B, Hu W, Dai L, Zhu P, Chang Z, Wu Q, Zhao Y, Jia Y, Xu P, Liu H, Shan G: Exon-intron circular rnas regulate transcription in the nucleus. Nat Struct Mol Biol 2015;22:256-264.

11 Salzman J, Gawad C, Wang PL, Lacayo N, Brown PO: Circular rnas are the predominant transcript isoform from hundreds of human genes in diverse cell types. Plos One 2012;7:e30733.

12 Chen LL, Yang L: Regulation of circrna biogenesis. RNA Biol 2015;12:381-388.

13 Suzuki H, Zuo Y, Wang J, Zhang MQ Malhotra A, Mayeda A: Characterization of rnase r-digested cellular rna source that consists of lariat and circular rnas from pre-mrna splicing. Nucleic Acids Res 2006;34:e63.

14 Suzuki H, Tsukahara T: A view of pre-mrna splicing from rnase r resistant rnas. Int J Mol Sci 2014;15:93319342.

15 Hansen TB, Jensen TI, Clausen BH, Bramsen JB, Finsen B, Damgaard CK, Kjems J: Natural rna circles function as efficient microrna sponges. Nature 2013;495:384-388.

16 Hansen TB, Kjems J, Damgaard CK: Circular rna and mir-7 in cancer. Cancer Res 2013;73:5609-5612.

17 Zhao J, Li L, Wang Q, Han H, Zhan Q, Xu M: Circrna expression profile in early-stage lung adenocarcinoma patients. Cell Physiol Biochem 2017;44:2138-2146.

18 Li H, Hao X, Wang H, Liu Z, He Y, Pu M, Zhang H, Yu H, Duan J, Qu S: Circular rna expression profile of pancreatic ductal adenocarcinoma revealed by microarray. Cell Physiol Biochem 2016;40:1334-1344.

19 Liu W, Zhang J, Zou C, Xie X, Wang Y, Wang B, Zhao Z, Tu J, Wang X, Li H, Shen J, Yin J: Microarray expression profile and functional analysis of circular rnas in osteosarcoma. Cell Physiol Biochem 2017;43:969-985. 


\section{Cellular Physiology Cell Physiol Biochem 2018;50:342-352 \begin{tabular}{ll|l} 
DOI: 10.1159/000494010 & Ond Biochemistry Publisned online:40ctober 2018 & $\begin{array}{l}\text { O 2018 The Author(s). Published by S. Karger AG, Basel } \\
\text { www.karger.com/cpb }\end{array}$
\end{tabular}}

Fan et al.: Circular RNA Expression and Laryngeal Cancer

20 Mao JR, Bristow J: The ehlers-danlos syndrome: on beyond collagens. J Clin Invest 2001;107:1063-1069.

21 Sanz-Pamplona R, Berenguer A, Cordero D, Mollevi DG, Crous-Bou M, Sole X, Pare-Brunet L, Guino E, Salazar R, Santos C, de Oca J, Sanjuan X, Rodriguez-Moranta F, Moreno V: Aberrant gene expression in mucosa adjacent to tumor reveals a molecular crosstalk in colon cancer. Mol Cancer 2014;13:46.

-22 Cui G, Yuan A, Goll R, Olsen T, Husebekk A, Vonen B, Florholmen J: Distinct changes of dendritic cell number and il-12 mrna level in adjacent mucosa throughout the colorectal adenoma-carcinoma sequence. Cancer Immunol Immunother 2007;56:1993-2001.

23 Jeck WR, Sharpless NE: Detecting and characterizing circular rnas. Nat Biotechnol 2014;32:453-461.

-24 Karsenty G, Park RW: Regulation of type i collagen genes expression. Int Rev Immunol 1995;12:177-185.

-25 Barcus CE, O’Leary KA, Brockman JL, Rugowski DE, Liu Y, Garcia N, Yu M, Keely PJ, Eliceiri KW, Schuler LA: Elevated collagen-i augments tumor progressive signals, intravasation and metastasis of prolactin-induced estrogen receptor alpha positive mammary tumor cells. Breast Cancer Res 2017;19:9.

26 Li J, Ding Y, Li A: Identification of col1a1 and col1a2 as candidate prognostic factors in gastric cancer. World J Surg Oncol 2016;14:297.

27 Hayashi M, Nomoto S, Hishida M, Inokawa Y, Kanda M, Okamura Y, Nishikawa Y, Tanaka C, Kobayashi D, Yamada S, Nakayama G, Fujii T, Sugimoto H, Koike M, Fujiwara M, Takeda S, Kodera Y: Identification of the collagen type 1 alpha 1 gene (col1a1) as a candidate survival-related factor associated with hepatocellular carcinoma. BMC Cancer 2014;14:108.

28 Humphries B, Yang C: The microrna-200 family: small molecules with novel roles in cancer development, progression and therapy. Oncotarget 2015;6:6472-6498.

29 Esquela-Kerscher A, Slack FJ: Oncomirs - micrornas with a role in cancer. Nat Rev Cancer 2006;6:259-269.

30 Yates LA, Norbury CJ, Gilbert RJ: The long and short of microrna. Cell 2013;153:516-519.

-31 Feng J, Sun Q, Wu T, Lu J, Qu L, Sun Y, Tian L, Zhang B, Li D, Liu M: Upregulation of atf-3 is correlated with prognosis and proliferation of laryngeal cancer by regulating cyclin d1 expression. Int J Clin Exp Pathol 2013;6:2064-2070.

32 Rothman KJ, Cann CI, Flanders D, Fried MP: Epidemiology of laryngeal cancer. Epidemiol Rev 1980;2:195209.

33 Kuper H, Boffetta P, Adami HO: Tobacco use and cancer causation: association by tumour type. J Intern Med 2002;252:206-224. 